\title{
Foaming binary solution mixtures of low molecular surfactant and polyelectrolyte
}

\author{
S. B. AIDAROVA \\ Al-Farabi Kazakh National University, Karasai Batyr St., 95A, Almaty, 480012, Kazakhstan; \\ Kazakh-Biritish Techinical University, 59 Tole bi St., Almaty, 480012, Kazakhstan \\ K. B. MUSABEKOV, Z. B. OSPANOVA \\ Al-Farabi Kazakh National University, Karasai Batyr St., 95A, Almaty, 480012, Kazakhstan \\ M. GUDEN* \\ Kazakh-Biritish Techinical University, 59 Tole bi St., Almaty, 480012, Kazakhstan; Mechanical \\ Engineering Department, Izmir Institute of Technology, Urla, Izmir, Turkey \\ E-mail: mustafaguden@iyte.edu.tr
}

Published online: 7 June 2006

The lifetime of water solution foams of sodium dodecylsulfate (DDS, low molecular weight surfactant) and sodium carboxymethylcellulose (SCMC, polyelectrolyte) and their binary mixtures was experimentally investigated. The effects of ionic strength and acidity on the foam life were also determined. In binary solutions, a synergic effect of DDS and SCMC on the surface tension reduction, most likely resulting from the interaction of the surfactant with polymer, was found. The addition of $\mathrm{NaCl}$ into solution or increasing the ionic strength was found to decrease the surface tension and reduce interfacial mobility, hence increased foam lifetime. The relatively low lifetime of binary solution foams in acidic medium was attributed to the reaction between SCMC and acid, which resulted in relatively small reductions in the viscosity and consequently lowered the solution viscosity. (c) 2006 Springer Science + Business Media, Inc.

\section{Introduction}

Foams, also known as cellular materials composing of liquid and air, are the class of materials characterized with their relatively low densities. The unique properties associated with their cellular structures in addition to their lightness have made them very attractive materials to be used in many different, diverse applications including washing and cleaning, construction, firefighting, ore enrichment by flotation, oil and gas industry, dust-depression and etc. [1-3]. The stable foam configurations with specific physical and chemical properties are needed in many of these and also for future applications of foams. The foam stabilization is therefore a key parameter for manufacturing foams with desired properties and involves many complex processes, of which the mechanisms are not completely understood. It is well known that high-molecular weight substances such as water-soluble polymers (WSPs) particularly polyelectrolytes (PEs) are the foam stabilizing elements [4-8]. The mechanisms of foam stabilization using WSPs however have not been completely clarified. This study is a further investigation of understanding the mechanisms of foam stabilization with water-soluble PEs with and without using a surfactant. The water solution foams containing sodium carboxymethylcellulose (SCMC) as polyelectrolyte and sodium dodecylsulfate (DDS), an anion active micelle forming surfactant, and their binary mixtures were studied in order to determine the effect solution composition, salt addition, ionic strength and acidity on the foam lifetime. Based on the experimental results, the possible effects such parameters on the foam lifetime were explained.

\section{Experimental}

Foams were prepared inside a foam column (height $=$ $20 \mathrm{~cm}$ and diameter $3 \mathrm{~cm}$ ) through air blowing into $12 \mathrm{ml}$ water solution of SCMC $\left(\left[\mathrm{C}_{6} \mathrm{H}_{7} \mathrm{O}_{2}(\mathrm{OH})_{3-m}\right.\right.$ $\left.\left(\mathrm{OCH}_{2} \mathrm{COOS}\right)_{m}\right]_{n}: n=580$, and $\left.m=1 \sim 3\right)$, DDS 


\section{SYNTACTIC AND COMPOSITE FOAMS}

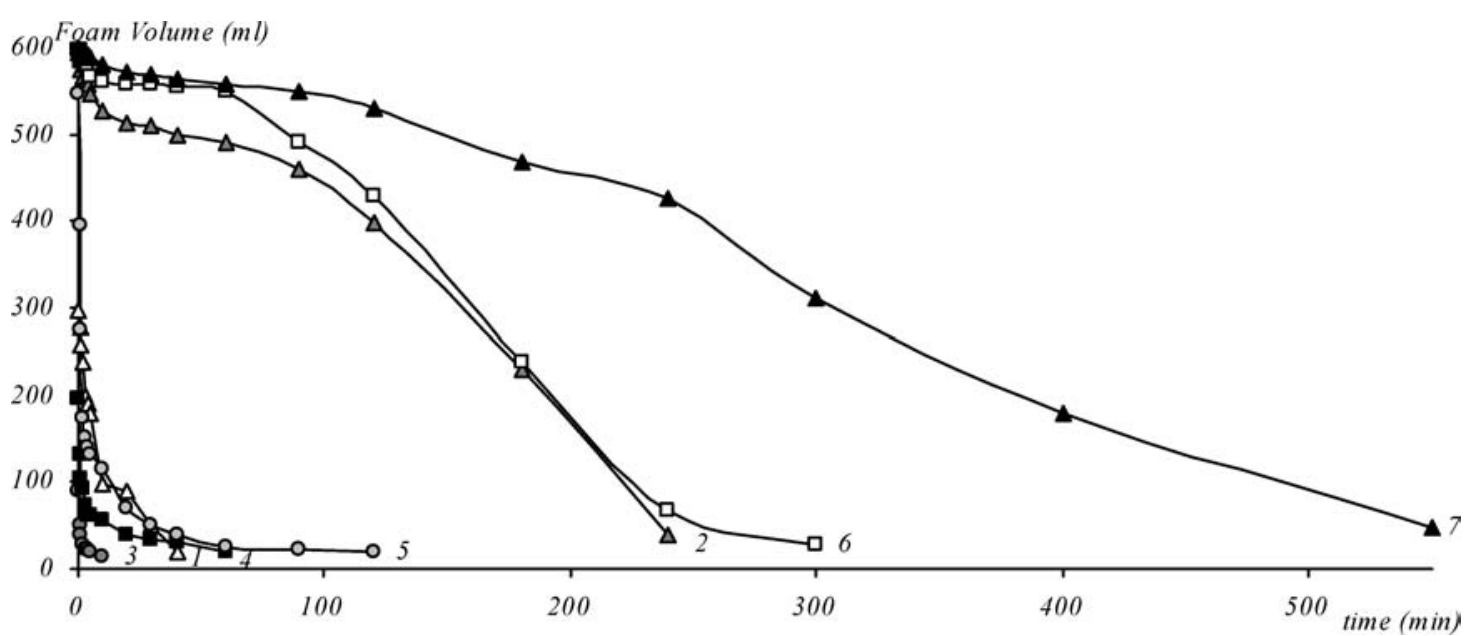

Figure 1 Foam volume vs. time: DDS solution with concentrations of $1 \times 10^{-3} \mathrm{~mol}^{-1}(1)$ and $1 \times 10^{-2}(2)$ and binary compositions ([SCMC] $=1 \times$ $10^{-2} \mathrm{~mol}^{-1}$ ) with $n$ values: 0.01 (3), $0.1(4), 0.25(5), 0.5(6)$ and 1 (7).

$\left(\mathrm{C}_{12} \mathrm{H}_{25} \mathrm{OSO}_{3} \mathrm{Na}\right)$ and their binary mixtures. Foaming was conducted at room temperature, $20^{\circ} \mathrm{C}$, for $40 \mathrm{~s}$. The volumes of prepared foams in the foam column were then measured as function of time in order to determine the foam collapse and foam stability. The first group of experiments was conducted on SCMC solutions (without DDS-addition) with concentrations ranging between $1 \times 10^{-6}$ and $1 \times 10^{-2} \mathrm{~mol} \mathrm{l}^{-1}$. In the second group, DDS solutions with concentration of $1 \times 10^{-3}$ and $1 \times 10^{-2} \mathrm{~mol} \mathrm{l}^{-1}$ were foamed without SCMC-addition. The foaming experiments were then continued with the binary mixtures of SCMS and DDS. In these experiments, the concentration ratio of DDS to SCMC, $n=\frac{[D D S]}{[S C M C]}$, ranged between $1 \times 10^{-4}$ and 1 and the concentration of SCMC was kept constant, $1 \times 10^{-2} \mathrm{~mol} \mathrm{l}^{-1}$. The capillary pressure of the foam plateau borders (Gibbs-Plateau) was measured using the porous plate technique [9]. In this technique the films were formed through a hole-drilled fritted glass disk that was connected to a capillary tube of a constant reference pressure. The equilibrium surface tensions of water solutions were determined by the Wilhelmy Plate method [10] with an accuracy of $\pm 0.2 \mathrm{mN} / \mathrm{m}$ at $25^{\circ} \mathrm{C}$ using a platinum plate. The viscosity of the solutions was measured using a capillary viscosimeter with a water expiration time of $113 \mathrm{~s}$. A photometer (KFK-3) was used to determine the optical densities of the solutions at a wave length of 490 nanometers.

\section{Results and discussions}

In the first group of experiments no foaming was observed within the investigated range of the SCMC concentrations $\left(1 \times 10^{-6}-1 \times 10^{-2} \mathrm{~mol} \mathrm{l}^{-1}\right)$. This would be expected since, as a polymer, SCMC only renders the foam stabilization by increasing the interface layer viscosity of the foam. The volume of the second group and binary mixture solution foams as function of time is shown in
Fig. 1. In the second group tests, the effect of increasing surfactant (DDS) concentration, from $10^{-3}$ (curve 1) to $10^{-2} \mathrm{~mol} \mathrm{l}^{-1}$ (curve 2), increases foam expansion. The foaming of DDS solutions was observed to start at concentrations above about $10^{-3} \mathrm{~mol} \mathrm{l}^{-1}$. On the contrary, the foaming of binary mixture solutions started at a DDS concentration as low as $10^{-4} \mathrm{~mol}^{-1}$ (curve 3 of Fig. 1). The increase of DDS concentration in binary solutions, as depicted in curves 4, 5, 6 and 7 of Fig. 1, further increases the foam expansion and improves the foam lifetime. The highest foam expansion and lifetime are found in the binary composition of $n=1$.

Fig. 2 shows the change of capillary pressures $(\Delta P \mathrm{~s})$ of the solutions in the foam column as function of time. The $\Delta$ Ps measurements were carried out until the maximum pressure reached. The foam solutions studied reach the equilibrium $\Delta P$ values within 10-15 min and the highest capillary pressure is shown by $10^{-2} \mathrm{~mol}^{-1}$ DDS solution (curve 1 of Fig. 2). Since DDS foam solution is comparably less stable and therefore collapses with a higher rate, it reaches the equilibrium $\Delta P$ value earlier. The lowest $\Delta P$ values, however, are found in the binary foam solutions as seen in Fig. 2. The equilibrium $\Delta P$ values also give qualitative information on the thicknesses of the foam films $[8,11]$. The foam collapse results from the film thinning process in which the liquid flows from the cell faces to the plateau borders under the influence of capillary pressure [12]. The relatively low values of $\Delta P$ measured in the binary foam solutions of DDS and SCMC confirms the above statement and is due to the reduced surface tension of the solutions with SCMC-addition leading to a decrease in the surface mobility. The surfaces of films are also likely to be saturated with DDS at increasing concentrations (near $n=1$ ), which predominantly determines the film properties. Therefore, the effect of SCMC-addition diminishes at DDS concentrations close to the critical micelle forming concentration. 


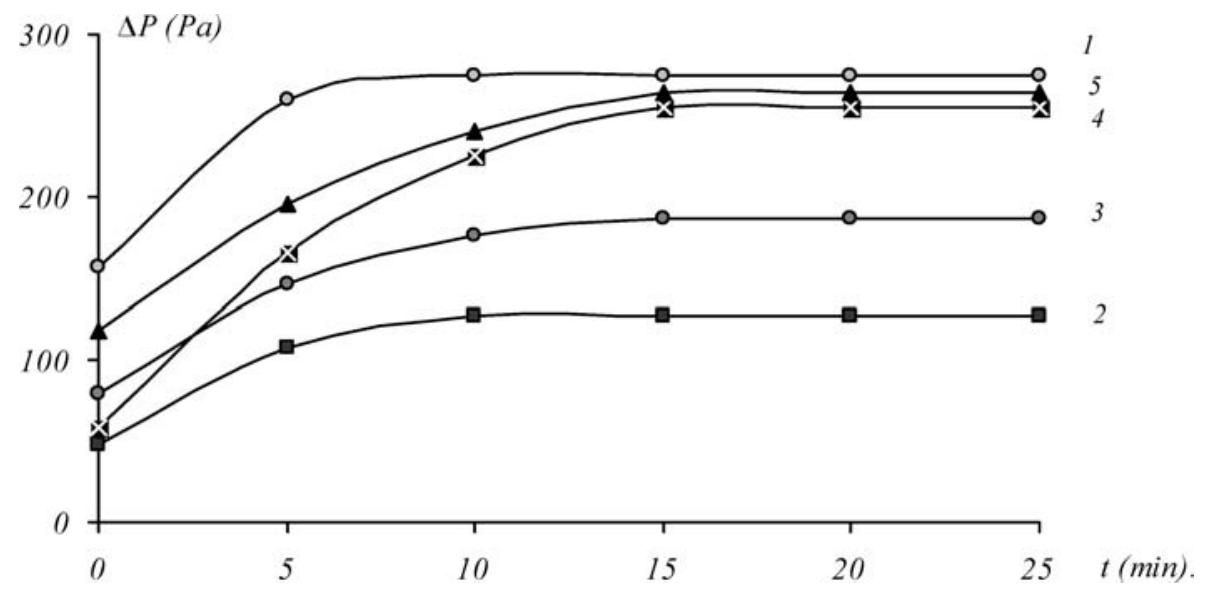

Figure 2 Capillary pressure vs. time: DDS solution with concentration $1 \times 10^{-2} \mathrm{~mol}^{-1}(1)$ and binary compositions $\left([\mathrm{SCMC}]=1 \times 10^{-2} \mathrm{~mol} \mathrm{1}^{-1}\right)$ with $n$ values 0.1 (2), $0.25(3), 0.5$ (4) and 1 (5).

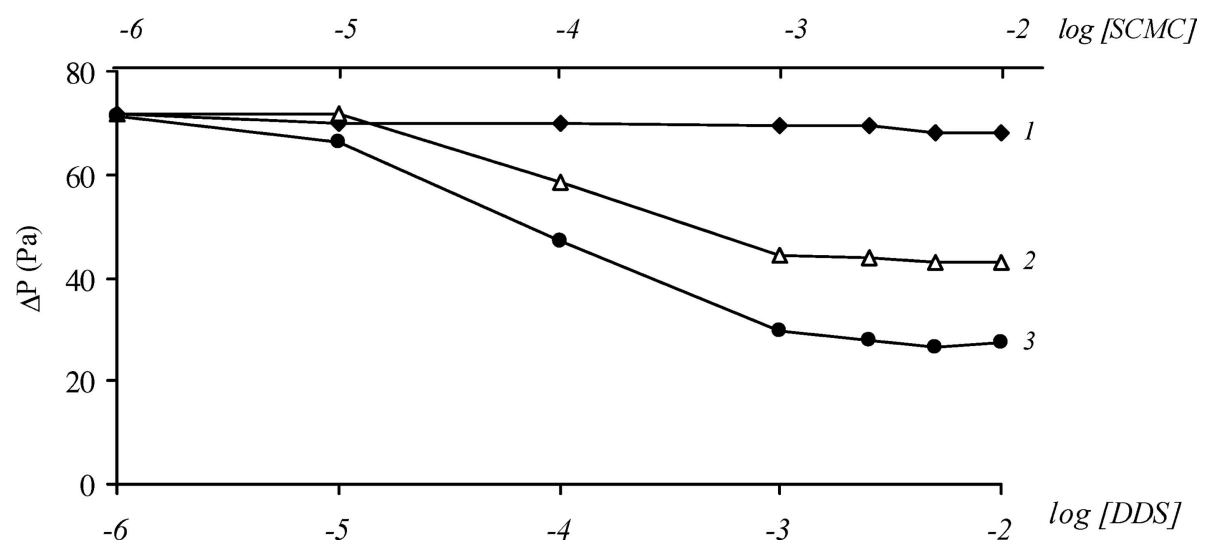

Figure 3 The isotherms of surface tension of water solutions as functions DDS and SCMC concentrations: SCMC solution (1), DDCS solution (2) and mixture solutions $\left([\mathrm{SCMC}]=1 \times 10^{-2} \mathrm{~mol}^{-1}\right)(3)$ at $t=25^{\circ} \mathrm{C}$.

The properties of the investigated foam solutions, the concentration ratio of DDS to SCMC $(n)$, maximum foam volume which is acquired after air bubbling to the solution for $40 \mathrm{~s}\left(V_{\max }\right)$, foam life $\left(\tau_{\min }\right)$ which is defined as the time at which $90 \%$ foam collapse occurs and equilibrium surface tension $(\sigma)$ are tabulated in Table I. It is clearly seen in this table that an increase in DDS concentration or $n$ value in binary solutions increases both $V_{\max }$ and $\tau_{\min }$.

Fig. 3 shows the effect of increasing concentrations of DDS and SCMC on the surface tension of binary foam solutions and the foam solutions containing only DDS and SCMC. Although increasing concentrations of DDS and SCMC decreases the surface tensions of solutions, the decrease in surface tension becomes more pronounced in the binary solutions containing the same concentrations of DDS and SCMC. In binary solutions; therefore, there exists a synergic effect of DDS and SCMC on the surface tension reduction, most likely resulting from the interaction of the surfactant with polymer [8]. The value of reduction in surface tension $\left(\Delta \sigma_{\text {synergy }}\right)$ for the binary

TABLE I Foam key parameters

\begin{tabular}{llllll}
\hline$[\mathrm{DDS}]\left(\mathrm{mol} \mathrm{1}^{-1}\right)$ & {$[\mathrm{SCMC}]\left(\mathrm{mol} \mathrm{l}^{-1}\right)$} & $n=\frac{[D D S]}{[S C M C]}$ & $V_{\max }(\mathrm{ml})$ & $\tau(\mathrm{min})$ & $\sigma\left(\mathrm{mN} \mathrm{m}^{-1}\right)$ \\
\hline $1 \times 10^{-4}$ & - & - & - & - & 58.37 \\
$1 \times 10^{-3}$ & - & - & 298 & 40 & 44.23 \\
$1 \times 10^{-2}$ & - & - & 593 & 240 & 40 \\
$1 \times 10^{-4}$ & $1 \times 10^{-2}$ & 0.01 & 90 & 60 & 47.87 \\
$1 \times 10^{-3}$ & $1 \times 10^{-2}$ & 0.1 & 196 & 120 & 29.70 \\
$2.5 \times 10^{-3}$ & $1 \times 10^{-2}$ & 0.25 & 546 & 300 & 27.87 \\
$5 \times 10^{-3}$ & $1 \times 10^{-2}$ & 0.5 & 598 & 550 & 26.33 \\
$1 \times 10^{-2}$ & $1 \times 10^{-2}$ & 1 & 600 & & 27.56 \\
\hline
\end{tabular}




\section{SYNTACTIC AND COMPOSITE FOAMS}

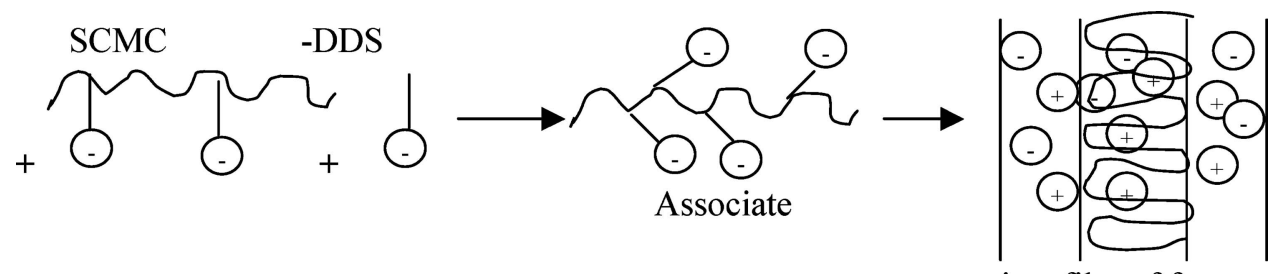

(a)

(b)

in a film of foam

(c)

Figure 4 The structure of interphase adsorption layer in foam films stabilized by surfactant and polymer.

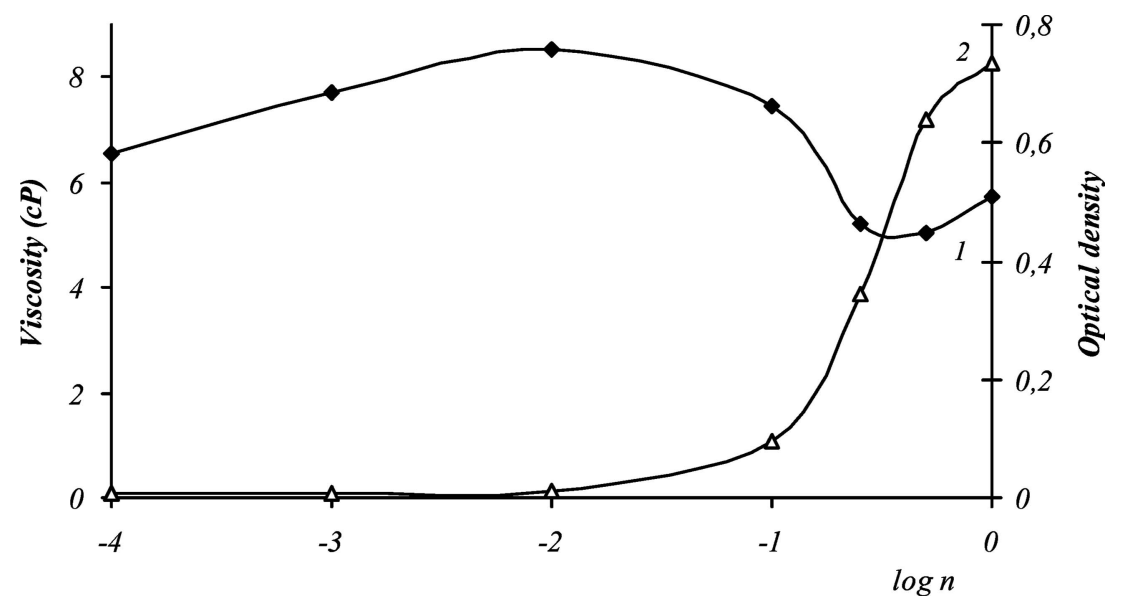

Figure 5 Viscosity (curve 1) and optical density (curve 2) of water solutions as function of logarithm of concentration ratio $n$ at $T=20^{\circ} \mathrm{C}([\mathrm{SCMC}]=$ $\left.1 \times 10^{-2} \mathrm{~mol}^{-1}\right)$.

solution of $n=1$ was determined using the methods described in $[13,14]$ and found $4.59 \mathrm{mN} \mathrm{m}^{-1}$.

The combined adsorption of polymer and surfactant occurs at the interfaces because of the interaction of polyelectrolyte with low-molecular surfactant polar groups. The low-molecular component is first adsorbed; then, the polymer forms a sub-layer, which determines the specific properties of interface adsorption layer [8]. It is therefore most likely that, the surface-active anions of low-molecular surfactant are first adsorbed (Fig. 4a) in the binary solution films. Then, negatively charged polyions are adsorbed on a sublayer due to hydrophobic interaction between DDS and SCMC (Fig. 4b). At an optimum DDS and SCMC concentration ratio, the polar groups are inverted into liquid phase in which the mixed adsorption layer forms a strong hydrophobic foam film (Fig. 4c). It is also possible that the foam stability is affected by the amplification of electrostatic interactions between the layers.

The variations of the viscosity and optical density of binary solutions as function of concentration ratio, $n$, are shown in Fig. 5. The viscosity initially increases with increasing $n$ until a maximum value corresponding to $n=$ $10^{-2}$. Further increase in $n$ value then results in decrease in the viscosity. Note also a sharp increase in the optical density corresponding to the maximum viscosity value $\left(n=10^{-2}\right)$ is clearly seen in Fig. 5; a consequence of the conformational transformations of macromolecules during the interaction with surfactant [13]. This also confirms a strong packing of macromolecules, resulting in reduction of SCMC molecules adsorption at the water-air interface. As a result such conformational transformations of macromolecules, their sites waterproofed by the bounded molecules of surfactant are braiding and forming original intermolecular micelles [15].

Foam stability can be affected by several factors including molecular packing in the adsorbed surfaces films and hence surface viscosity of the film at the air/water interface [16] and layering and or structuring micelles within the bulk water within the film (bulk viscosity) [17]. Fig. 6 shows the effect of polymer concentration (SCMC addition) at a constant concentration of DDS $\left(1 \times 10^{-3} \mathrm{~mol} \mathrm{l}^{-1}\right)$ on the foam stability. The stability of the foam monotonically increases with the increase of SCMC concentration as shown in Fig. 6 and it is possible to form long steady foam at high enough polymer concentrations. DDS foam solutions viscosity although increases with increasing SCMC concentration addition; however, it is not a dominant factor for stabilization. The considerable increase in viscosity was found only at relatively high polymer concentrations, but foam stabilization was noticeable at considerably lower polymer concentrations 


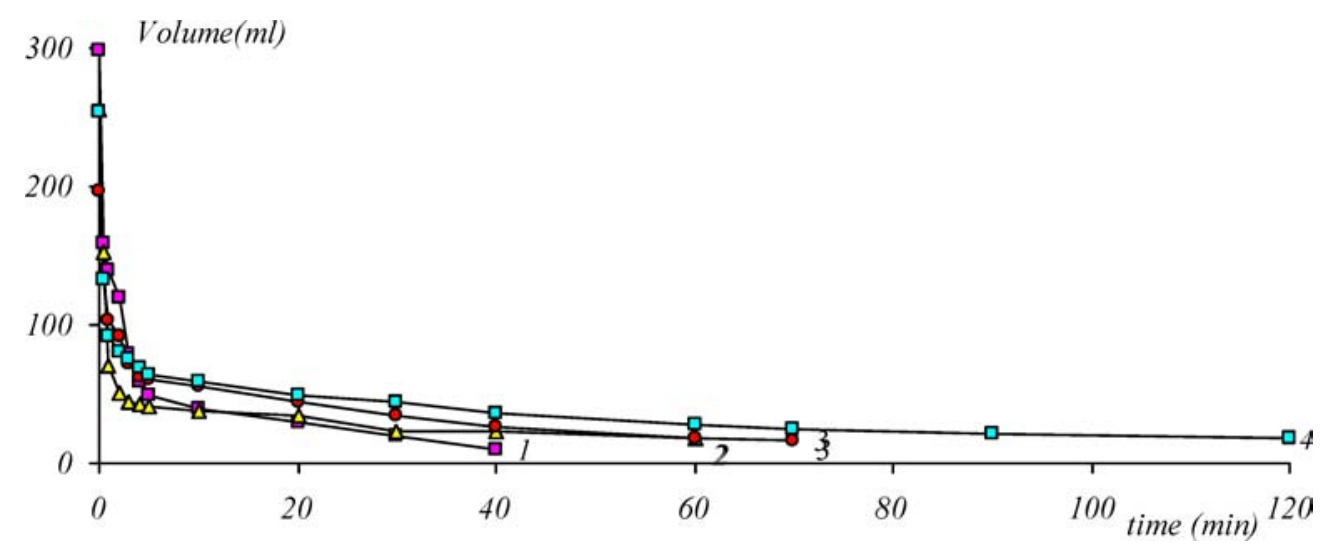

Figure 6 Volume vs. time for DDS foams of $10^{-3} \mathrm{~mol}^{-1}$ with SCMC concentrations of 0 (curve 1), $3 \times 10^{-3}$ (curve 2), $1 \times 10^{-2}$ (curve 3 ) and $5 \times$ $10^{-2} \mathrm{~mol} \mathrm{1}^{-1}$ (curve 4).

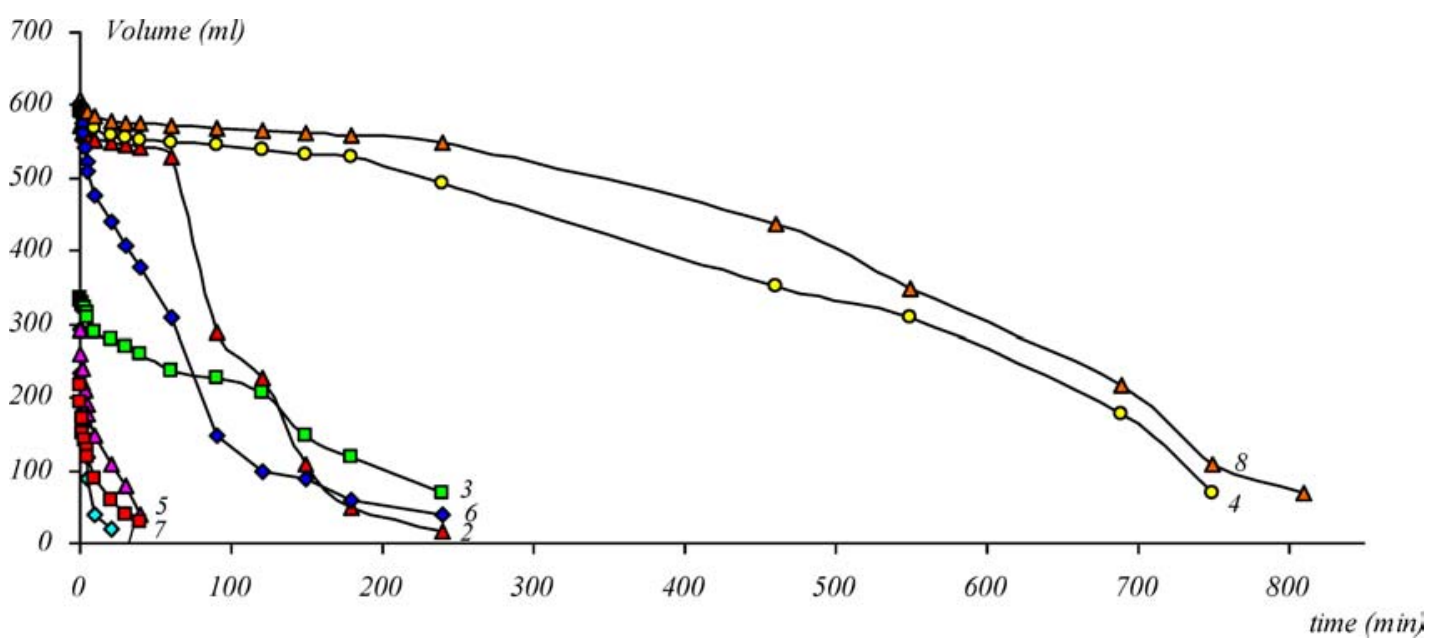

Figure 7 Foam volume vs. time of DDS and binary solutions at ionic strengths of (a) $0.01 \mathrm{~N}$ (curves-1, 2, 3 and 4) and (b) $0.1 \mathrm{~N} \mathrm{NaCl}$ (curves-5, 6, 7 and 8). DDS solution concentartions: $1 \times 10^{-3}(1), 1 \times 10^{-3}(5), 1 \times 10^{-2}(2)$ and $1 \times 10^{-2}(6)$ Binary compositions $(n): 0.1(3), 0.1(7), 1(4), 1(8)$ with $[\mathrm{SCMC}]=1 \times 10^{-2} \mathrm{~mol} \mathrm{l}^{-1}$.

(Fig. 6, curve 3). Therefore, it may be concluded that a major contribution to foam stabilization is due to the formation of a mixed adsorption-layer of DDS and SCMC, causing a more tightly packed adsorbed surface layer and hence higher surface viscosity.

It is well known that inorganic electrolytes such as potassium and sodium chlorides and sodium phosphates improve the foam forming ability of detergent materials $[1,2]$. It is shown in Fig. 7, the addition of sodium chloride $(\mathrm{NaCl})$ into DDS solution also increases the foam lifetimes (Fig. 7, curves 1, 2, 5, 6). NaCl-addition into DDS both increases the viscosity of liquid, causing slow drainage of the water in the films and the adsorption density of anions at the air/water solution interface. The increased foam lifetime with $\mathrm{NaCl}$-addition was attributed to the decrease of hydrophobic force in the film [18]. Fig. 8 shows the isotherms of surface tension and foam lifetime of aqueous solution of DDS and SCMC mixtures with different ionic strengths of $\mathrm{NaCl}$-addition. The increasing of ionic strength form 0.01 to $0.1 \mathrm{H}$ results in a decrease in foam lifetime as seen in Fig. 8 (curves 4 and 5) at high $n$ value (1), which was most likely due to screening effect, resulting in decrease of electrostatic repulsion in the foam films [18].

The effect of solution $\mathrm{pH}$ on the foam lifetimes of DDS and binary solutions is shown in Fig. 9. It is known that, in anionic surfactant solutions, the lifetime of foams increases with increasing acidity [2]. A similar effect is also observed in the investigated solutions. In DDS solutions, increasing acidity increases the foam lifetime (curves 1 and 3 in Fig. 9), while binary solution foams show increased lifetime in alkaline conditions (curves 4 and 6 in Fig. 9). It should also be noticed that for each solution investigated, there may exist an optimum acidity at which the lifetime is maximized. The dependency of surface tension of DDS, SCMC and binary solutions to acidity was investigated and the results are shown in Fig. 10. In binary solutions, it is clearly seen that a higher surface tension reduction occurs at lower $\mathrm{pH}$ values (Fig. 10, curves 3, 4, $5)$. 


\section{SYNTACTIC AND COMPOSITE FOAMS}

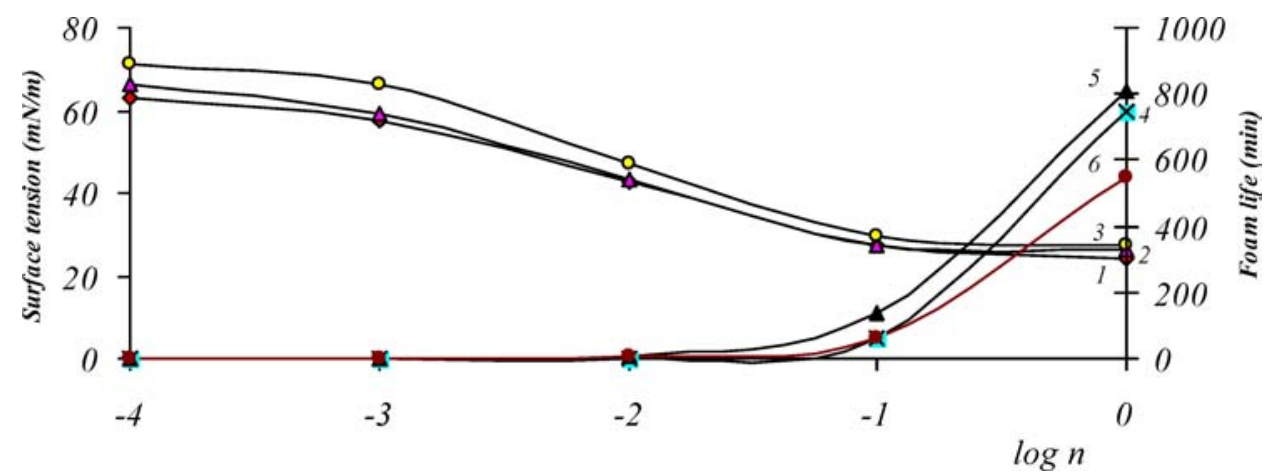

Figure 8 Surface tension (1-3) and foam life (4-6) vs. $\log n$ of binary compositions of DDS and SCMC solutions at (a) $0.1 \mathrm{H}$ (1 and 4), (b) $0.01 \mathrm{H}$ (2 and 5) and (c) water solution (3 and 6) with $[\mathrm{SCMC}]=1 \times 10^{-2} \mathrm{~mol}^{-1}\left(T=25^{\circ} \mathrm{C}\right)$.

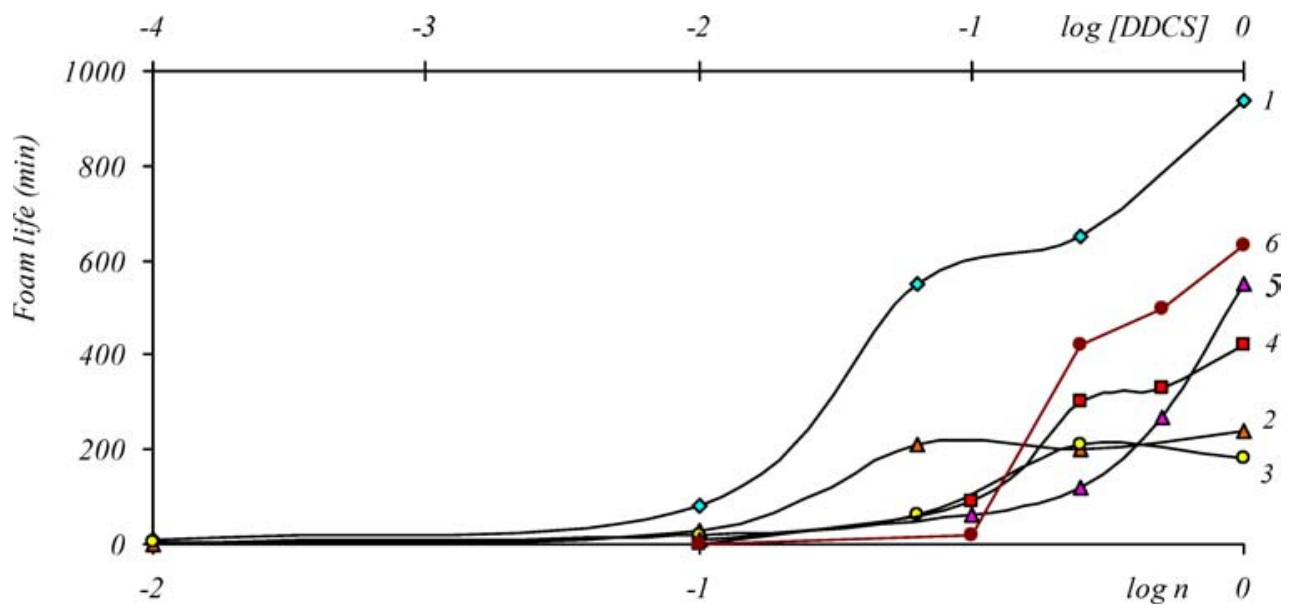

Figure 9 Foam life vs. [DDCS] (curves 1-3) and $n$ by (curves 4-6) at different $\mathrm{pH}$ values: $\mathrm{pH}=2$ (curves 1 and 4), $\mathrm{pH}=7$ (curves 2 and 5) and $\mathrm{pH}=$ 12 (curves 3 and 6).

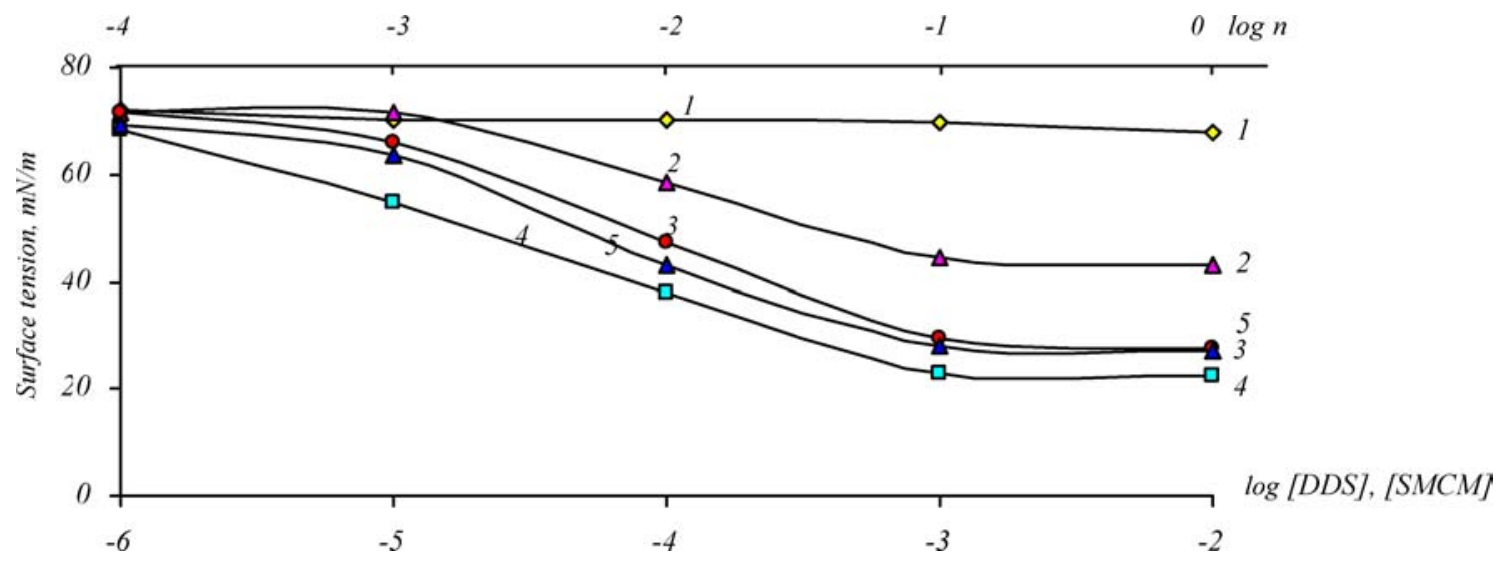

Figure 10 Surface tension of aqueous solutions as function of [SCMC] (1), [DDS] (2), $n(3,4,5)$; and $\mathrm{pH}(\mathrm{pH}=7(3), \mathrm{pH}=2(4)$ and $\mathrm{pH}=12(5))$.

The electrostatic factor plays a critical role in the foam stability enchancement with the addition of electrolytes. At lower electrolyte concentrations, a dense salty adsorption layer forms waterproofing at the gas-liquid interface, promoting the foam forming ability and foam stability. At increasing salt concentrations; however, the concen- tration of surfactant decreases in the adsorption layer due to precipitate formation; therefore, reducing both foaming ability and foam stability $[19,20]$. P.A. Rebinder was the first who marked the relation between the saturation rate of adsorptive layer with the surfactant and the stability of foams received from these solutions. It was shown that 


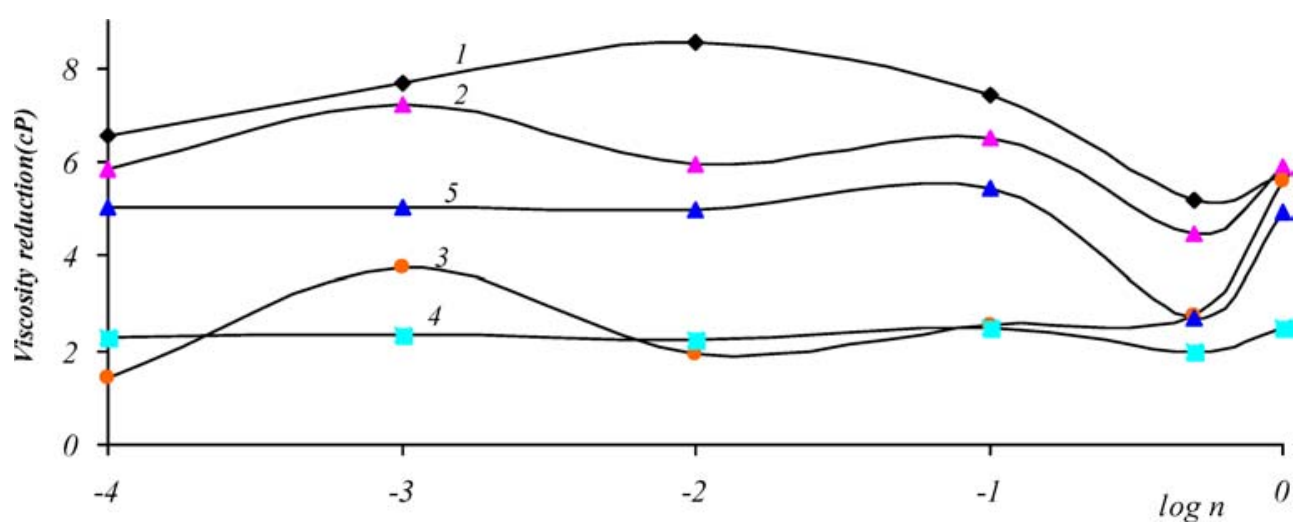

Figure 11 Viscosity reductions of binary solutions of varying $\mathrm{n}$ values with $\mathrm{NaCl}$-addition and acidity: $\mathrm{pH}-7$ (1), $0.01 \mathrm{~N} \mathrm{NaCl}(2), 0.1 \mathrm{~N} \mathrm{NaCl}(3), \mathrm{pH}-2$ (4) and $\mathrm{pH}-12$ (5). [SCMC] $=1 \times 10^{-2} \mathrm{~mol}^{-1}$.

the saturation of an adsorptive layer reached at surfactant concentrations in the presence of electrolytes. It also results in the formation of more steady foams than foams of distilled water with the same concentrations of surfactant $[19,20]$.

The viscosity reduction in binary solutions (the mechanical factor of stability of foam stabilization) with $\mathrm{NaCl}$-addition and acidity are shown in Fig. 11 for varying $n$ values. The highest reductions in viscosity are found at $\mathrm{pH}-7$ (1) and $12(5)$ and in the solution of $0.01 \mathrm{~N}$ $\mathrm{NaCl}$-addition (2). As is seen in Fig. 11, NaCl-addition (2 and 3 ) and acidity (4 and 5) have similar effects on the viscosity reductions, although viscosity reduction values are different. The relatively lower foam lifetime of binary solution foams in acid medium was further attributed to the reaction between SCMC and acid, which resulted in relatively smaller reductions in viscosity.

This study has clearly shown that the foam lifetime of binary foam solutions of polyelectrolyte and low molecular weight surfactant can be established as function of composition, ionic strength and acidity. The maximum foam lifetime was determined by the degree of linkage of polymer to surfactant at the expense of hydrophobic interplays. Also, the addition of $\mathrm{NaCl}$ to DDS-SCMC solution may potentially lead to production of new composite foams for the fire extinguishing in marine crude oil and gas production areas.

\section{Conclusions}

The lifetime of water solution foams with DDS (low molecular weight surfactant) and SCMC (polyelectrolyte) and their binary composition was investigated. The effects of ionic strength (with $\mathrm{NaCl}$-addition) and acidity on the foam lifetime were also determined. The increased viscosity of the binary solutions with SCMC-addition increased the foam lifetime. In binary solutions, a synergic effect of DDS and SCMC on the surface tension reduction, most likely resulting from the interaction of the surfactant with polymer, was found. In DDS solutions, increasing acidity increased the foam lifetime, while binary solution foams showed higher lifetime in alkaline conditions. The relatively lower lifetimes of binary solution foams in acid medium was attributed to the reaction between SCMC and acid, which resulted in relatively smaller reductions in viscosity. The addition of $\mathrm{NaCl}$ into solution was found to decrease the surface tension and surface mobility hence increased foam lifetime.

\section{References}

1. P. M. KRUGLJAKOV and D. R. EKSEROVA, "Foam and Foamy Films" (Moscow Chemistry, Moscow, 1990).

2. V. K. Tikhomirov, "Foam" (Moscow Chemistry, Moscow, 1975).

3. S. B. AJDAROVA, K. B. MUSABEKOV, K. Z. ABDIE and R. IDRISOVA, 2nd Regional Scientific and Technical Conference (Karaganda, Kazakhstan, 1985) p. 32.

4. S. B. BAKESHEVA, S. B. AIDAROVA and N. N. ZAMAIN, Bull. KazSU 11 (1998) 44.

5. N. G. VILKOVA, T. N. HASAKOVA and P. M. KRUGLIAKOV, Coll. Chem. 57 (1995) 783.

6. V. N. IZMAJLOVA, S. R. DERKACH, G. P. JAMPOLSKAJA, K. V. ZOTOVA and V. N. CHERnin, Coll. Chem. 59 (1997) 654.

7. V. G. BABAK, G. A. VIHOREVA, I. G. LUKINA and L. V. KUZNETS OVA, ibid. 59 (1997) 149.

8. S. B. AIDAROVA S. B. PhD. Thesis (1992).

9. K. J. MYSELS and M. N. JONES, Farad Soc. 42 (1966) 42.

10. L. I. OSIPOW, "Surface Chemistry: Theory and Industrial Applications" (Krieger, Huntington, NY, 1977) p. 20.

11. A. A.TRAPEZNIKOV, Some films, foams properties and questions of their stability, Foams. Reception and application. Materials of All-Union scientific and technical conference-the Part 1 (M.: the Science, 1974) p. 137.

12. B. V. DERIAGIN, "Stability Theory of Colloids and Thin Films" (M: the Science, 1986) p. 240.

13. K. B. MUSABEKOV, K. A. ZHUBANOV, V. N. IZMAILOVA and B. D. SUM, "Polyelectrolyte Interphase Layers" (Alma-Ata: Science, 1987) p. 151.

14. K. B. MUSABEKOV, S. B. AIDAROVA and K. Z. A B DIEV, "Successes of Colloid Chemistry" (L.: Chemistry, 1991) p. 209.

15. C. BARAN, Coll. Chem. 64 (2002) 591. 


\section{SYNTACTIC AND COMPOSITE FOAMS}

16. B. JONNSON, B. LINDMAN, K. HOLMBERG and B. KRONBERG, "Surfactants and Polymers in Aqueous Solutions" (Wiley, NY, 1998).

17. A. D. NIKOLOV and D. T. WASSAN, J. Coll. Inter. Sci. 133 (1989) 1.

18. L. WANG and R.-H. YOON, Langmuir 20 (2004) 11457.
19. N. I. GONCHARIK, F. F. MOZHEIKO and C. A. GOLUBOVICH, Influence of non-organic electrolytes on foam forming ability of sodium salts of carbon acids (Vesting NAN Beloruss, Chemical Scientist Edition, 1991) p. 91.

20. B. T. CHISTYAKOV and B. H. CHERNIN, "Education and Foam Stability" (Chemistry Advances, 1991) p. 91. 